\title{
Specific heat of a particle on the cone
}

\author{
E. S. Moreira, Jr* $*$ and E. S. Oliveira \\ Instituto de Ciências Exatas, Universidade Federal de Itajubá, \\ Av. BPS 1303 Pinheirinho, 37500-903 Itajubá, MG, Brazil
}

(Dated: March 21, 2006)

\begin{abstract}
This work investigates how a conical singularity can affect the specific heat of systems. A free nonrelativistic particle confined to the lateral surface of a cone - conical box - is taken as a toy model. Its specific heat is determined as a function of the deficit angle and the temperature. For a vanishing deficit angle, the specific heat is that of a particle in a flat disk where a characteristic temperature separates quantum and classical behaviors, as usual. By increasing the deficit angle the characteristic temperature increases also, and eventually another characteristic temperature (which does not depend on the deficit angle) arises. When the cone gets sufficiently sharp, at low and intermediate temperatures the azimuthal degree of freedom is suppressed. At low temperatures the specific heat varies discontinuously with the deficit angle. Connections between certain theorems regarding common zeros of the Bessel functions and this discontinuity are reported.
\end{abstract}

PACS numbers: 03.65.Ge, 02.30.Gp, 05.20.-y, 05.30.-d

\section{INTRODUCTION}

As techniques in condensed matter physics to fabricating very thin samples with various topologies and geometries develop, the study of quantum mechanics on curved surfaces becomes increasingly relevant. Among these backgrounds a cone is perhaps the one with the simplest nontrivial geometry - it is flat everywhere except at its tip where there is a conical singularity $[1,2,3,4,45$. Due to local flatness the conical singularity does not affect local dynamics; but it does affect global features such as the solutions of differential equations. One is dealing, therefore, with a geometric analog of the Aharonov-Bohm setup [6].

There has appeared a considerable number of works on quantum mechanics of a particle in conical spaces, since the early investigations in Refs. 7, $8,[9]$. Nevertheless, it seems that a study on how a conical singularity affects the statistical mechanics of a particle has not yet been pursued. It should be mentioned that since physics around a topological defect in the bulk of a solid can be seen as physics in an effective conical geometry [10], the study of statistical mechanics in conical spaces may help to learn about thermal properties when topological defects are present.

This paper addresses quantum statistical mechanics of a free nonrelativistic particle on the cone. The focus is on the specific heat $C$. In Sec. II the classical partition function is calculated, showing that classical $C$ is not affected by the conical singularity. In Sec. III, the particle energy spectrum is obtained and an analysis on how it depends on the deficit angle is presented. In particular, connections with properties of the zeros of the Bessel functions are discussed. In the following section, the energy spectrum is used to obtaining the quantum specific heat. Quantum $C$ is then studied at low, intermediate and high temperatures, showing that the conical singularity can affect it substantially. In Sec. $\mathrm{V}$ the paper is closed with a summary and final remarks.

\section{CLASSICAL STATISTICAL MECHANICS}

One begins by defining the background (a more detailed account on the conical geometry can be found for example in Refs. [5, 11]). A wedge of angle $\mathcal{D}$ is removed from a disk of radius $a$, and the two opposite sides of the missing wedge are identified, resulting a cone of deficit angle $\mathcal{D}$. It is convenient to express $\mathcal{D}$ in terms of the cone parameter $0<\alpha \leq 1$,

$$
\mathcal{D}=2 \pi(1-\alpha)
$$

Then a flat disk (i.e., absence of conical singularity) corresponds to $\alpha=1$, and the cone becomes increasingly sharper as $\alpha$ decreases from unity. It is worth mentioning that a cone with $\mathcal{D}=\pi$ corresponds to the configuration space of

*Electronic address: moreira@unifei.edu.br 
a system of two identical particles (here separate by a maximum distance $a$ ) with respect to its centre of mass [12.

A free norelativistic particle of mass $M$ in thermodynamical equilibrium with a reservoir at temperature $T$ is constrained to move on the cone described above. The corresponding Hamiltonian is given by

$$
\mathcal{H}=\frac{p_{\rho}^{2}}{2 M}+\frac{p_{\varphi}^{2}}{2 M \rho^{2}}
$$

where $p_{\rho}$ and $p_{\varphi}$ are the usual momenta associated with polar coordinates $\rho$ and $\varphi$. Although Eq. (2) carries no trace of the conical singularity [Eq. (2) is simply the Hamiltonian of a free particle on the Euclidean plane], the latter appears in the identification

$$
(\rho, \varphi+2 \pi \alpha) \sim(\rho, \varphi) .
$$

It can be shown that the conical singularity at $\rho=0$ corresponds to an integral curvature proportional to $\mathcal{D}[4]$.

Observing Eq. (2), the standard procedure to compute the (semi) classical partition function by integrating over the phase space [13],

$$
Z_{(2)}=\frac{1}{h^{2}} \int_{0}^{a} d \rho \int_{0}^{2 \pi \alpha} d \varphi \int_{-\infty}^{\infty} d p_{\rho} \int_{-\infty}^{\infty} d p_{\varphi} e^{-\mathcal{H} / \kappa T}
$$

yields

$$
Z_{(2)}=\frac{A}{h^{2}} 2 \pi M \kappa T
$$

where

$$
A:=\alpha \pi a^{2}
$$

is the area of the cone. Once a partition function $Z$ is known, the internal energy is given by

$$
U=\kappa T^{2} \frac{1}{Z} \frac{d Z}{d T}
$$

As the dependence of $Z_{(2)}$ on $\alpha$ happens only through a temperature independent factor, the internal energy and, consequently, the specific heat

$$
C=\frac{d U}{d T}
$$

are clearly not affected by the conical singularity. Indeed, these formulae give

$$
U=\kappa T \quad C=\kappa
$$

as one might have guessed by evoking the equipartition theorem at an early stage [see Eq. (22)]. [In fact, as a straightforward covariant calculation can show, the results in Eqs. (5) and (9) hold for any two-dimensional curved box regardless its topology and geometry.]

\section{ENERGY SPECTRUM}

In order to use the tools of quantum statistical mechanics, one needs to know the energy spectrum of the system - a free particle in a conical box. Considering Eq. (3) the stationary states $\Psi$ must satisfy

$$
\Psi(\rho, \varphi+2 \pi \alpha)=\Psi(\rho, \varphi) .
$$

Eq. (2) leads to a Hamiltonian operator which is simply $-\hbar^{2} / 2 M$ times the flat Laplacian written in polar coordinates. One then faces the task of solving the eigenvalue problem for the flat Laplacian operator on the cone $[\underline{8}$, [9], with the additional boundary condition

$$
\Psi(\rho=a, \varphi)=0
$$


since the particle is confined to a circular box of radius $a$. (This is essentially the eigenvalue problem which appears in the study of vibrations of a circular membrane [14].)

Up to a normalization constant, the regular solutions (i.e., finite at $\rho=0$ ) are

$$
\Psi=J_{|n| / \alpha}\left(k_{n, s} \rho\right) \exp \{i n \varphi / \alpha\} \quad k_{n, s}:=\frac{1}{a} \chi_{|n| / \alpha, s}
$$

where $n$ is an integer, $s$ is a positive integer, and $\chi_{\nu, s}$ denotes the $s$ th positive zero of the Bessel function of the first kind $J_{\nu}(x)$ 14, 15, 16, 17]. Approximated values for the zeros can be obtained by truncating the expansion [14]

$$
\chi_{\nu, s}=\left(\frac{\nu}{2}+s-\frac{1}{4}\right) \pi-\frac{4 \nu^{2}-1}{8 \pi}\left(\frac{\nu}{2}+s-\frac{1}{4}\right)^{-1}-\ldots
$$

at some point which depends on the accuracy required. Eq. (11) is specially adequate in dealing with high zeros (large $s$ ).

Then, for a pair of quantum numbers $(n, s), \Psi$ is a state with angular momentum $n \hbar / \alpha(n>0$ and $n<0$ correspond to the particle winding counterclockwise and clockwise, respectively) and energy

$$
E_{n, s}=\frac{\hbar^{2} k_{n, s}^{2}}{2 M}
$$

both quantities, therefore, discrete. As $E_{n, s}=E_{-n, s}, E_{n \neq 0, s}$ is at least doubly degenerate (degeneracy will be addressed in more detail below).

In order to have a picture of the energy spectrum, and of the corresponding stationary states, one recalls that $J_{\nu}(x)$ is an oscillatory function of $x$ and that its zeros $\chi_{\nu, s}$ are labelled such that $\chi_{\nu, 1}<\chi_{\nu, 2}<\chi_{\nu, 3}<\ldots$. It is also important to know that $\chi_{\nu, s}$ is a continuous increasing function of $\nu[15]$. Then $E_{n, s} \geq E_{0,1}$, i.e.,

$$
E_{0,1}=\frac{\hbar^{2}}{2 M}\left(\frac{2.4048 \ldots}{a}\right)^{2}
$$

is the zero point energy $\left(\chi_{0,1}\right.$ has been replaced by its numerical value [16]), and $E_{n, s}$ increases with $|n| / \alpha$ and $s$. Clearly, energy levels with $n=0$ are not affected by the conical singularity (even classically the conical singularity only affects the motion if the particle has nonvanishing angular momentum [1]]).

To figure out the way that $\alpha[\mathcal{D}$, cf. Eq.(11)] shapes the energy spectrum, it helps to know that for $\alpha=1$ (particle confined to a flat disk [18]) the energy spectrum resembles that of a particle in a square box of the same area, although this analogy should not be pushed too far (e.g., the degeneracies of the corresponding energy levels do not match completely). When $\nu>0, \chi_{\nu, s}>\nu$ [17], leading to

$$
E_{1,1}>\frac{\hbar^{2}}{2 M a^{2} \alpha^{2}} .
$$

Since $E_{1,1}$ is the energy of the first excited state with $n \neq 0$, as the cone gets sharper (i.e., as $\alpha$ decreases from unity) all the energy levels with nonvanishing angular momentum are swept up in the spectrum, leaving those with vanishing angular momentum unchanged. It should be mentioned that for large $\nu, \chi_{\nu, 1} \simeq \nu+1.8557 \nu^{1 / 3}[16]$, resulting that ">" in Eq. (14) can be replaced by " $\simeq$ " when $\alpha$ is small, with increasing accuracy as $\alpha \rightarrow 0$.

In order to discuss degeneracy of the energy levels, it is appropriate to begin with $\alpha=1$. As two Bessel functions of the first kind with integer orders cannot have common zeros (this follows from a theorem known as Bourget's hypothesis [15]), it results that energy levels with $n=0$ are not degenerate, whereas those with $n \neq 0$ are doubly degenerate. This situation changes when the conical singularity is present $(\alpha \neq 1)$, leading to a new source of degeneracy, since the Bessel functions in Eq. (10) may have common zeros (see Ref. [19] and references therein).

Noting that $\chi_{0,1}<\chi_{1,1}<\chi_{2,1}<\chi_{0,2}<\ldots$ [16], when $\alpha=1$ Eq. (12) yields

$$
E_{0,1}<E_{1,1}<E_{2,1}<E_{0,2}<\ldots
$$

As $\alpha$ decreases, $E_{1,1}$ and $E_{2,1}$ increase, whereas $E_{0,1}$ and $E_{0,2}$ remain the same. Eventually $E_{2,1}$ catches $E_{0,2}$ up, and the energy level $E_{0,2}=E_{2,1}$ becomes triply degenerate. By further lowering $\alpha$, the configuration in Eq. (15) evolves to

$$
E_{0,1}<E_{1,1}<E_{0,2}<E_{2,1}<\ldots
$$


Carrying on with the process, as $\alpha$ reaches the value (numerically determined)

$$
\alpha_{o}=0.4338 \ldots,
$$

Eq. (16) changes to

$$
E_{0,1}<E_{1,1}=E_{0,2}<E_{2,1}<\ldots
$$

where the energy level $E_{0,2}=E_{1,1}$ is again triply degenerate. When $\alpha<\alpha_{o}$, Eq. (17) is replaced by

$$
E_{0,1}<E_{0,2}<\ldots<E_{1,1}<\ldots
$$

According to Eq. (14) and the discussion that follows it, as $\alpha \rightarrow 0$ more and more levels $E_{0, s}$ appear on the left of $E_{1,1}$ in Eq. (18), resulting in that the lower part of the energy spectrum corresponds to states with vanishing angular momentum.

These considerations are relevant to finding the behavior of the specific heat, as will be seen in the next section.

\section{SPECIFIC HEAT}

In quantum statistical mechanics [13] the internal energy is given by Eq. (7) with the partition function now obtained by summing over the stationary states discussed in the previous section,

$$
Z=\sum_{n=-\infty}^{\infty} \sum_{s=1}^{\infty} \exp \left\{-E_{n, s} / \kappa T\right\}
$$

As usually happens, a closed expression (in terms of known analytic functions) is not available for the summations in Eq. (19), and a combination of analytical and numerical procedures is required to deal with them. It is convenient to define two characteristic temperatures, namely:

$$
\Theta_{\alpha}:=\frac{E_{1,1}-E_{0,1}}{\kappa} \quad \Theta:=\frac{E_{0,2}-E_{0,1}}{\kappa}
$$

It follows from the discussion in Sec. III that $\Theta_{\alpha}$ increases as $\alpha$ decreases from unity, $\Theta_{\alpha=\alpha_{o}}=\Theta$, and as $\alpha \rightarrow 0$,

$$
\Theta_{\alpha} \simeq \frac{\hbar^{2}}{2 M a^{2} \kappa \alpha^{2}}
$$

As seen in the previous section, the degree of degeneracy of the first excited energy level is 2,3 or 1 , for $\alpha>\alpha_{o}$, $\alpha=\alpha_{o}$ and $\alpha<\alpha_{o}$, respectively. Taking this fact to Eq. (19), Eqs. (7) and (8) yield for the low temperature behavior $(T \rightarrow 0)$ of the specific heat $C(\alpha)$,

$$
C\left(\alpha>\alpha_{o}\right) \simeq 2 \kappa\left(\frac{\Theta_{\alpha}}{T}\right)^{2} e^{-\Theta_{\alpha} / T} \quad C\left(\alpha=\alpha_{o}\right) \simeq 3 \kappa\left(\frac{\Theta}{T}\right)^{2} e^{-\Theta / T} \quad C\left(\alpha<\alpha_{o}\right) \simeq \kappa\left(\frac{\Theta}{T}\right)^{2} e^{-\Theta / T}
$$

where the integers multiplying $\kappa$ are the degree of degeneracy just mentioned. Eq. (22) shows that $C$ varies discontinuously with $\alpha$ when $T$ is much smaller than the characteristic temperatures in Eq. (20). As $T$ increases, higher energy levels have to be considered in Eq. (19), and Eq. (22) no longer holds.

The way that $C$ departs from Eq. (22) is shown in Fig. 1 In particular, for small values of $\alpha$ the ways that $C$ departs from $C\left(\alpha<\alpha_{o}\right)$ in Eq. (22) are approximately the same. This can be understood by recalling that when $\alpha$ is small the lower part of the spectrum is essentially formed by energy levels $E_{0, s}$ which do not depend on $\alpha$. Eq. (21) shows that for small $\alpha$ the azimuthal characteristic temperature $\Theta_{\alpha}$ is high, and consequently the various plots of $C$ split from each other only at higher temperatures, when the populations of energy levels with nonvanishing angular momentum become appreciable.

At the regime $T \ll \Theta_{\alpha}$ with $\alpha<\alpha_{o}\left[\alpha \geq \alpha_{o}\right.$ necessarily leads to the corresponding low temperature behavior in Eq. (22)], only states with $n=0$ effectively counts in Eq. [19], resulting

$$
Z \simeq \sum_{s=1}^{\infty} \exp \left\{-E_{0, s} / \kappa T\right\} .
$$


By setting $\nu=0$ in Eq. (11), Eq. (12) yields

$$
E_{0, s}=\frac{h^{2}}{8 M a^{2}}\left(s-\frac{1}{4}\right)^{2}+\ldots,
$$

which up to the term $1 / 4$ and the corrections is the energy of a free particle in a one-dimensional box of length $a$ (and this identification improves as $s$ increases). As $T \rightarrow 0$, Eq. (223) leads to $C\left(\alpha<\alpha_{o}\right)$ in Eq. (22). When $\alpha$ is sufficiently small such that

$$
\Theta \ll T \ll \Theta_{\alpha}
$$

applies, the summation in Eq. (23) can be replaced, in first approximation, by an integration over $s$ from 1 to $\infty$, with $E_{0, s}$ given by Eq. (24) [one can safely truncate Eq. (24) at the first term to obtain the main contribution]. Using for example Refs. 16] or [20], evaluation of the integration yields $Z \simeq Z_{(1)}$, where

$$
Z_{(1)}=\frac{a}{h} \sqrt{2 \pi M \kappa T}
$$

is the classical partition function of a particle in a one-dimensional box of length $a$. Eqs. (7) and (8) then give $U \simeq \kappa T / 2$ and

$$
C \simeq \frac{\kappa}{2}
$$

showing that the conical singularity indeed suppresses one degree of freedom [cf. Eq. (92)].

With $\alpha$ smaller than about 0.1 , Fig. [ 1 shows that $C$ gets a maximum value just above $\kappa / 2$, at $T$ close to $\Theta$ (see Fig. 1 for more accurate values). If $\Theta \ll \Theta_{\alpha}$ also holds, the regime $T \ll \Theta_{\alpha}$ resembles the thermal behavior of the specific heat of a particle in a one-dimensional box of length $a$ [21].

When the temperature is much larger than both the characteristic temperatures, one can proceed as above and replace the summations in Eq. (19) by integrations. Noting Eqs. (10) to (12), and performing the integration over $s$ [16, 20] leads to an integration over $n$ of a complementary error function, namely

$$
\int_{0}^{\infty} \operatorname{erfc}(x) d x=\frac{1}{\sqrt{\pi}}
$$

which has been evaluated by regularizing related formulae in Refs. 16, 17. The leading contribution in $Z$ is proportional to the classical partition function in Eq. (5), with the proportionality constant depending on where Eq. (11) is truncated: keeping only the first term, and then also the second term, results

$$
Z \simeq 0.81 Z_{(2)} \quad Z \simeq 0.94 Z_{(2)}
$$

respectively (in fact, to obtain the second expression one has to evaluate an additional integration involving the complementary error function [20]). Using either results in Eq. (28), Eqs. (7) and (8) yield as leading contribution

$$
C \simeq \kappa
$$

which is the classical value, as expected. [The discrepancies in Eq. 28) and its failure in reproducing $Z_{(2)}$ exactly can be understood by noticing that for large $n$ in Eq. (19), terms beyond the first term in Eq. (11) become relevant.]

A maximum also appears, now above $\kappa$, at a temperature comparable to $\Theta_{\alpha}$, as shown in Fig. 1 In particular the plot for $\alpha=1$ (particle in a flat disk) resembles that of a particle in a square box of the same area [21].

\section{CONCLUSION}

Motivated by the fact that statistical mechanics of systems in atomic size boxes is very sensitive to the topology and geometry of the boxes, this work treated the specific heat $C$ of a free nonrelativistic particle in a conical box. The energy spectrum was determined and its features were obtained from properties of the zeros of the Bessel functions. In particular, degeneracy of the energy levels was related to long established theorems regarding common zeros of the Bessel functions.

This toy model exposed with clarity the effects of a conical singularity on $C$ at various regimes of temperature. At low temperatures $C$ varies discontinuously with the deficit angle [cf. Eq. (22)]. At low and intermediate temperatures, 


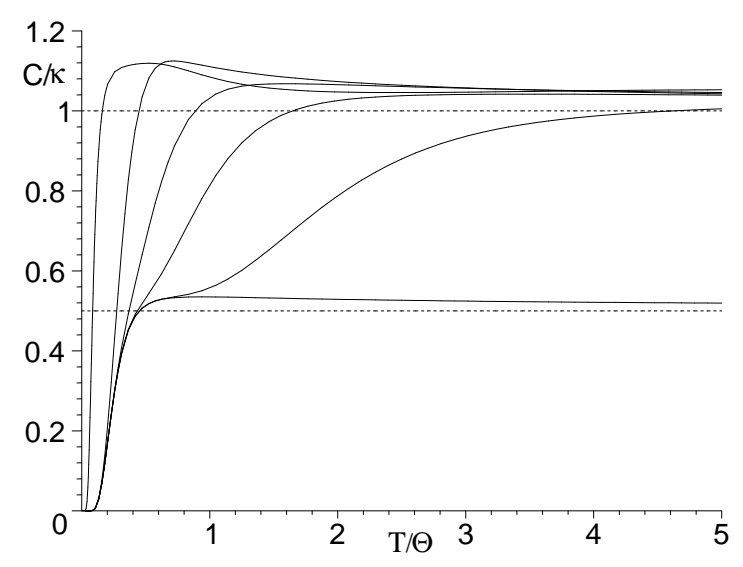

FIG. 1: Numerical plots of $C / \kappa$ versus $T / \Theta$. The plots from the left correspond to $\alpha=1,0.3,0.2,0.15,0.1$ and $\alpha \rightarrow 0$, respectively. The plots for $\alpha=1$ to $\alpha=0.15$ display maxima corresponding to specific heat of about $1.119 \kappa, 1.125 \kappa, 1.068 \kappa$ and $1.042 \kappa$ at temperatures of about $0.52 \Theta, 0.72 \Theta, 1.59 \Theta$ and $3.49 \Theta$, respectively. At $T \simeq 0.94 \Theta$, the plot for $\alpha \rightarrow 0$ displays a maximum corresponding to $C \simeq 0.535 \kappa$.

if the cone is sufficiently sharp, the azimuthal degree of freedom is suppressed [cf. Eq. [27)]. At high temperatures the radial and the azimuthal degrees of freedom are fully excited, leading to the value of $C$ prescribed by the equipartition theorem [cf. Eq. (29)]. The numerical plots in Fig. 1illustrate these facts.

The effect of the sharpness of the cone on $C$ can be better appreciated by contrasting the two limits $\alpha=1$ and $\alpha \rightarrow 0$. When $\alpha=1$, the energy spectrum is that of a free particle in a flat disk, where energy levels with vanishing and nonvanishing angular momentum are mixed up along the spectrum. As temperature $T$ rises, the radial and the azimuthal degrees of freedom get equally excited, leading to the high temperature value $C \simeq \kappa$, which is consistent with the equipartition theorem. This picture changes radically as one sets $\alpha \rightarrow 0$. When the limit $\alpha \rightarrow 0$ is literally taken, the energy of the first excited state with nonvanishing angular momentum gets infinitely high in the spectrum. Then, as $T$ increases, only the radial degree of freedom gets progressively excited, resulting in $C \simeq \kappa / 2$ at higher temperatures - the $\alpha \rightarrow 0$ limit suppresses the azimuthal degree of freedom.

This work also aimed to tune mathematical tools in quantum statistical mechanics on the cone such that other thermodynamical quantities, as well as more elaborate models, can be addressed. Some remarks are in order.

The regular solutions in Eq. (10) are not the only ones consistent with square integrability of the wave function and conservation of probability 22, 23. . A mild divergence at the origin (where the conical singularity sits) does not spoil these physical requirements [1]]. It seems that the choice for regular solutions relies in that they arise naturally when the conical singularity is "smoothed" by regularization [22].

The discontinuity of the specific heat in Eq. (22) is a feature of any thermal system whose degree of degeneracy $g$ of the first excited energy level $\epsilon_{1}$ varies with some parameter. If $\epsilon_{o}$ is the zero point energy, as $T \rightarrow 0$, a quick calculation shows that

$$
C \simeq g \kappa\left(\frac{\theta}{T}\right)^{2} e^{-\theta / T}
$$

where $\theta:=\left(\epsilon_{1}-\epsilon_{o}\right) / \kappa$. This kind of discontinuity at low temperatures also appears, for example, in the specific heat of the anisotropic rigid rotator [24].

Recalling that even the main contributions in Eq. (28) were affected by where one truncates Eq. (11), the determination of corrections in Eqs. (27) and (29) turns out to be a rather tricky task. Although the leading contributions in Eqs. (27) and (29) do not depend on where Eq. (11) is truncated (either on the lower limit of the related integrations), this may not be the case when dealing with their corrections. Such a problem is somewhat similar to that where two distinct approximations for the energy spectrum give the same leading contribution in the expression for the high temperature behavior of $C$; but different corrections (e.g., the anharmonic oscillator in Ref. [25]). Therefore, in order to check correctness at every step in using Eq. (11) to finding subleading contributions, a thorough numerical analysis must be implemented (it should be noted that this is the approach in Ref. [25]).

Since the disk and the cone have the same topology [11], the suppression of one degree of freedom in Eq. (27) is a geometrical rather than a topological effect - it is due to a curvature singularity.

Although the background addressed in this work is a cone, for which $\alpha$ is not greater than unity [cf. Eq. (10], it 
should be pointed out that in the context of topological defects in solids [10] there is room for $\alpha>1$ in the effective geometry corresponding to disclinations. In this case, as $\alpha$ increases from unity, $E_{1,1}$ approaches $E_{0,1}$ and $\Theta_{\alpha}$ in Eq. (20) decreases, suggesting that the classical limit in Eq. (29) would be reached at lower temperatures (this point needs further investigation though).

\section{Acknowledgments}

The authors are grateful to Renato Klippert and Ricardo Medina for their assistance on computational matters. This work was partially supported by the research agencies CNPq and FAPEMIG.

[1] L. Marder, Proc. R. Soc. London, Ser. A 252, 45 (1959).

[2] A. Staruszkiewicz, Acta Phys. Pol. 24, 734 (1963).

[3] J. S. Dowker, Nuovo Cimento B 52, 129 (1967).

[4] D. D. Sokolov and A. A. Starobinskii, Dok. Akad. Nauk 234, 1043 (1977) [Sov. Phys. Dokl. 22, 312 (1977)].

[5] S. Deser, R. Jackiw and G. 't Hooft, Ann. Phys. (N.Y.) 152, 220 (1984).

[6] V. B. Bezerra, J. Math. Phys. 30, 2895 (1989).

[7] J. S. Dowker, J. Phys. A 10, 115 (1977).

[8] G. 't Hooft, Commun. Math. Phys. 117, 685 (1988); S. Deser and R. Jackiw, ibid 118, 495 (1988); P. S. Gerbert and R. Jackiw, ibid 124, 229 (1989).

[9] D. Lancaster, Ph.D. thesis, Stanford University (1984); Phys. Rev. D 42, 2678 (1990).

[10] E. Kröner, in Continuum Theory of Defects - Physics of Defects, Proceedings of the XXXV Less Houches Session, 1980, edited by R. Balian et al. (North-Holland, Amsterdam, The Netherlands, 1981); M. O. Katanaev and I. V. Volovich, Ann. Phys. (N.Y.) 216, 1 (1992); F. Moraes, Braz. J. Phys. 30, 304 (2000); M. O. Katanaev, Phys. Usp. 48, 675 (2005).

[11] E. S. Moreira, Jr., Phys. Rev. A 58, 1678 (1998).

[12] J. M. Leinaas and J. Myrheim, Nuovo Cimento 37B, 1 (1977).

[13] R. K. Pathria, Statistical Mechanics (Pergamon Press, Oxford, 1972).

[14] A. Gray and G. B. Mathews, A Treatise on Bessel Functions and Their Applications to Physics (Dover Publications, New York, 1966).

[15] G. N. Watson, A Treatise on the Theory of Bessel Functions (Cambridge University Press, Cambridge, 1944).

[16] M. Abramowitz and I. A. Stegun, Handbook of Mathematical Functions (Dover Publications, New York, 1965).

[17] I. S. Gradshteyn and I. M. Ryzhik, Table of Integrals, Series, and Products (Academic Press, New York, 1965).

[18] R. W. Robinett and S. Heppelmann, Phys. Rev. A 65, 062103 (2002).

[19] E. N. Petropoulou, P. D. Siafarikas and I. D. Stabolas, J. Comp. Appl. Math. 153, 387 (2003).

[20] A. P. Prudnikov, Yu. A. Brychkov and O. I. Marichev, Integrals and Series (Gordon and Breach Science Publishers, Amsterdam, 1986).

[21] H. B. Rosenstock, Am. J. Phys. 30, 38 (1962).

[22] B. S. Kay and U. M. Studer, Commun. Math. Phys., 139, 103 (1991); M. Bourdeau and R. D. Sorkin, Phys. Rev. D 45, 687 (1992).

[23] G. Bonneau, J. Faraut and G. Valent, Am. J. Phys. 69, 322 (2001); D. A. Konkowski, T. M. Helliwell and C. Wieland, Class. Quantum Grav. 21, 265 (2004).

[24] A. O. Caride and C. Tsallis, J. Stat. Phys. 35, 187 (1984).

[25] A. M. Mariz and C. Tsallis, Phys. Rev. A 29, 2871 (1984). 